\title{
Emotions can alter kinesthetic acuity
}

Léonard Samain-Aupic, Rochelle Ackerley, Jean-Marc Aimonetti, Edith Ribot-Ciscar*

Aix Marseille Univ, CNRS, LNSC (Laboratoire de Neurosciences Sensorielles et Cognitives UMR 7260), Marseille, France.

* Corresponding author: Laboratoire de Neurosciences Sensorielles et Cognitives (UMR 7260), Centre Saint-Charles Pôle 3C - Case B, Aix-Marseille Université - CNRS, 3 place Victor Hugo 13331, Marseille, Cedex 03, France, edith.ribot-ciscar@univ-amu.fr

\begin{abstract}
Kinesthesia, the perception of our own body movements, relies on the integration of proprioceptive information arising mostly from muscle spindles, which are sensory receptors in skeletal muscles. We recently showed that emotions alter the proprioceptive messages from such muscle afferents, making them more sensitive to muscle lengthening when participants were listening sad music. Presently, we investigated whether these changes in proprioceptive feedback relating to emotional state may affect the perception of limb movements. Kinesthetic acuity was tested in 20 healthy, young adults by imposing rampand-hold movements that consisted of either plantar flexion or dorsiflexion movements of the ankle at $0.04 \%$, or no movement. These were imposed during four emotional conditions (listening to neutral, sad, or happy music, or no music). The participants were asked to relax and focus on music (or nothing), and then they shifted their focus to the direction of an incoming movement. Once this had finished, they were asked its direction. Muscle activity, heart rate, and electrodermal activity were recorded during each trial, and after each music condition the participants rated the emotion felt on a visual analog scale. The rating of the emotional content of the music corroborated with changes in physiological measures. Kinesthetic acuity was also affected by the emotional state and found to be larger during the sad condition, as compared to the no music or neutral conditions. We conclude that emotion can shape our perception of movements, which we show here where feeling sadness significantly increase our kinesthetic acuity, this may be functionally relevant for the preparation of appropriate behavioral responses.
\end{abstract}




\section{Highlights}

- We investigated whether emotions alter the perception of limb movements

- Emotions were modulated by music and kinesthetic acuity was tested at the ankle

- Kinesthetic acuity increased particularly in the sad condition

- Emotions increase our movement sense which may aid appropriate behavior

Keywords: acuity, emotions, kinesthesia, muscle afferents, movement sense, proprioception

\section{Introduction}

In the past two decades, studies have shown that emotions shape the content of our percepts and awareness [1], yet often, emotions are not taken into account during perceptual tasks. Emotions can modulate our perception directly, where relevant stimuli are favored, to allow the individual to cope with a situation. This has been particularly shown in the visual and auditory modalities [2]. To our knowledge, the question as to whether this heightening of perceptual responses applies to bodily information has not been investigated; however, proprioception, our ability to sense the location and movement of body parts, is of major importance in behavioral situations [3]. For example, patients lacking proprioceptive information cannot hold a steady joint angle, nor or adapt their movements to an unexpected load, without visual input $[4,5]$. Such patients also show deficits in multi-joint movements and the trajectory of their movement is imprecise, even when they have visual feedback [6].

Proprioceptive afferent information is mostly provided by muscle spindles, sensory receptors found in the muscles, whose sensitivity may be modulated by the central nervous system $[7,8]$. There exists an efferent system to specifically control the stiffness of the muscle spindles, i.e. the gamma fusimotor system, making the receptors more or less responsive to changes in muscle length $[7,8]$. Recently, we demonstrated that the encoding of body movements by muscle spindles depends on the emotional context, by altering the top down control of muscle spindle sensitivity [9]. These results were obtained via microneurography, a technique which allows the recording of axonal unitary activity in sensory fibers in superficial nerves in humans [10]. In our former study, sensory recordings were made from 
muscle spindles relating to the ankle joint and participants' emotional state was manipulated through listening to evocative classical music, to induce sad, neutral, or happy emotions; these music pieces have been previously shown to activate emotional areas in the brain [11]. Specifically, in these conditions, we found that muscle afferent responses to ankle movements were consistently increased in the sad condition as compared to neutral. The happy condition gave rise to less clear-cut effects in the whole sample of muscle afferents, since it either increased or decreased the movement response, depending on the participant.

These effects have been interpreted in the frame of the well-documented influence of emotion on motor activities, such as during erect posture [12], isometric contractions [13], gait [10], or the readiness to move [14], which are all impacted by the emotional context. Muscle spindle activities are involved in the regulation of motor activities [15], where increases in their firing may influence future movement by directly enhancing the excitability of motoneurons in the spinal cord [16]. It is well known that pleasant experiences prime approach actions and unpleasant experiences prime withdrawal [17], and the increased muscle spindle activity may contribute to such emotional motor reactions. However, since muscle spindle messages are of major importance in the knowledge of our posture and movement, of the whole body or parts of it, one may hypothesize that any change in spindle messages may also impact the perception of limb movements; the present study was undertaken to investigate this hypothesis.

\section{Material and methods}

\section{Participants}

20 healthy young adults ( $24 \pm 6$ years, 10 females) participated in the study. All participants gave their written, informed consent and the study was approved by the local ethics committee (Comité de Protection des Personnes Sud-Méditerranée I).

\section{Experimental set-up}

The participants were seated comfortably in an armchair with their legs positioned in cushioned grooves so that a standardized, relaxed position could be maintained, without muscle activity. The knee joint was at an angle of approximately $120-130^{\circ}$. Their right foot 
was laid on a stationary plate, and the left foot was laid on a rotating pedal connected to a computer-controlled machine that permitted the imposition of ramp-and-hold ankle movements. Muscle activity was monitored throughout the experiments by recording surface electromyographic activity (EMG). Two pairs of surface electrodes were placed over the Gastrocnemius Soleus (GS) and Tibialis Anterior (TA) muscle bellies. The EMGs were recorded with a high gain $(10,000)$, band-pass filtered $(30-300 \mathrm{~Hz})$, and sampled at $5 \mathrm{kHz}$. Measures of emotional responses were obtained through recording electrodermal activity, using two surface electrodes placed on each side of the left hand (gain: $x$ 500, band-pass: 0.1-100 Hz, sampling frequency: $200 \mathrm{~Hz}$ ), and heart rate, recorded by means of a passive transducer, strapped around the left index finger. The EMG, electrodermal activity, and heart rate measures were monitored throughout the experiment.

\section{Manipulation of emotional state}

The participant's emotional state was modified by listening to classical music through headphones (Bose; Framingham, MA). The music pieces had been previously defined as reliably inducing sad ( $n=9$ pieces), happy $(n=7)$, or neutral $(n=5)$ affective states (see [9] for details regarding the music pieces). Before the experiment started, the participant listened to the pieces of music and rated each of them on a visual analog scale (VAS), ranging from sad (equating to 0 , the saddest they could feel) to happy (equating to 10 , the happiest they could feel). For each participant, the most effective music in triggering each type of emotion was chosen to be played during the psychophysiological experiment (see [11]).

\section{Kinesthetic acuity}

To test kinesthetic acuity, we used a protocol validated in a previous study (see [18]). In brief, ramp-and-hold movements of a constant angular velocity $\left(0.04^{\circ} / \mathrm{s}\right)$ were imposed at the ankle joint, and the participants were asked to report the movement direction. The initial position of the ankle was slightly plantar-flexed $\left(100^{\circ}\right)$, and either a dorsal flexion or a plantar flexion was imposed. Participants provided their response after completion of the movement during the hold phase, which lasted for $3 \mathrm{~s}$. The foot was then returned to its initial position (Fig. 1). A session was composed of 5 dorsiflexions, 5 plantar flexions, and 5 equal time-windows without any movement (called no-movement), all in a random order. During each session, the participants kept their eyes closed. 


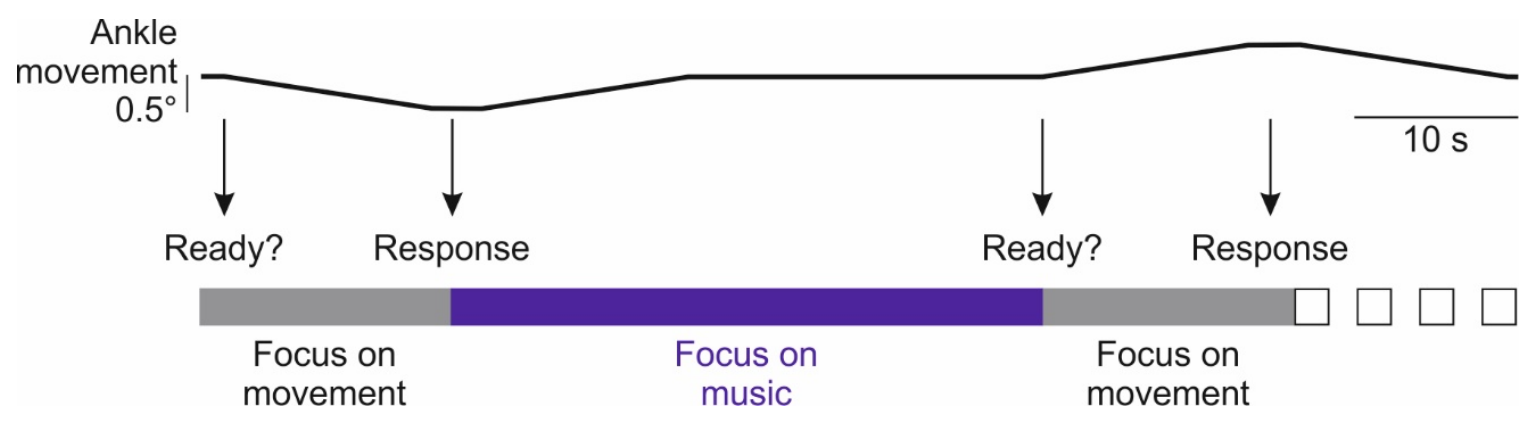

Figure 1: Experimental paradigm for the proprioceptive task.

The figure shows an example timeline of the course of the kinesthetic experiment. The participant was instructed that the movement would begin and then that they needed to focus on the movement. After, the participant gave an oral response and focused on the music. This was repeated for the different levels of movement and over the conditions.

Participants were previously instructed to relax, to focus on the music, and then to shift their focus on the movement as soon as a 'Ready?' trigger was delivered (see Fig. 1). The participant focused on listening to the music for half a minute on average, which occurred between the response and start of the next movement. Participants were asked to report the direction of any movement they perceived just prior to the end of the hold phase and only when they could report it with certainty. Independent of the participant's performance, the instructions were frequently repeated to minimize the number of incorrect responses.

\section{Experimental protocol}

Prior to the experiment, the participants were familiarized with the task and their movement sub-threshold amplitude was established. Here, the movement was initially imposed with an amplitude set at $0.6^{\circ}$ (see [19]). This amplitude was considered the participant's subthreshold if she/he correctly reported the direction of four to six of ten successive movements. When fewer or more correct responses were given, we increased or decreased the movement amplitude by $0.1^{\circ}$, respectively.

After, the four recording sessions (sad, happy, neutral, no music), were performed in a random order. No feedback regarding the participant's performance was given. Each session lasted for approximately 15 mins and these sessions were separated by 5 min rest periods 
during which the participant was allowed to move his/her feet. After each session, the participant was asked to rate the emotion felt for the music on a further VAS, which was used in the analysis. The duration of the entire experiment was approximately $2 \mathrm{~h}$.

\section{Data analysis}

The kinesthetic acuity data were expressed as the percentage of correct responses. A correct response corresponded to the reporting of the actual movement direction or of the correct absence of movement $[\%$ correct $=($ Ncorrect $/$ Ntotal $) * 100$, with Ntotal $=15]$. When the participant was uncertain of his/her response, the response was qualified as 'undetermined'. When participant reported the wrong direction, or reported a movement when no movement was imposed, these were considered as false-positive responses. As well as comparing between the different conditions, we expressed the acuity values as compared to the neutral condition, in order to eliminate the potential effect of simply listening to music and to keep the effect of the valence of emotion as the only factor.

All the acuity, rating, and physiological data were analyzed using non-parametric statistics (due to violations in normality, as found using Shapiro-Wilk tests, and due to the use of percentage and ordinal data) in Prism (version 6; GraphPad, La Jolla, CA). Friedman ANOVA tests were used to compare between the different conditions, per measure, and Dunn's multiple comparison corrected post-hoc tests were used to compare between conditions. Significances are given to 3 decimal places.

\section{Results}

The participants were asked to report the direction of any movement they perceived only when they could report it with certainty. This aim was achieved, as the rate of false-positive responses was $27 \%$ for the all sessions.

A significant main effect was observed for the subjective emotional ratings generated by the music (Friedman ANOVA $=39.52, p<0.001$ ), where sad was significant rated as the lowest (significant difference between sad and neutral, Dunn's test $p=0.004$ ) and happy was rated 
as the highest (significant difference between neutral and happy, Dunn's test $p=0.008$ ) (Fig. 2A).

(A) Emotion rating

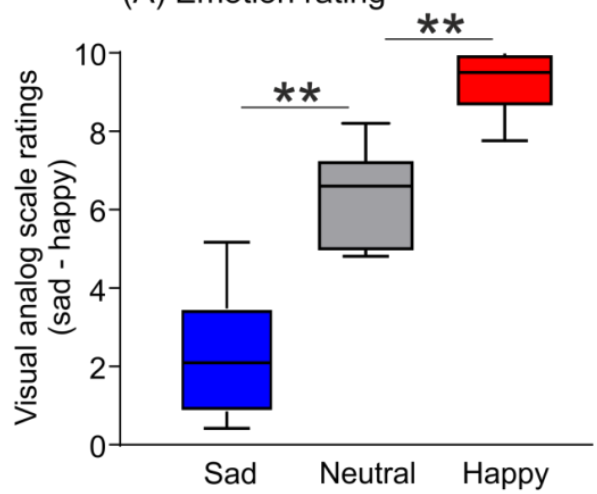

(C) Heart rate

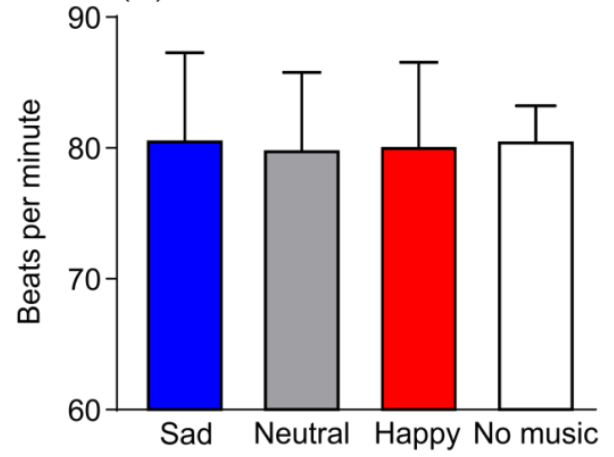

(E) EMG - GS

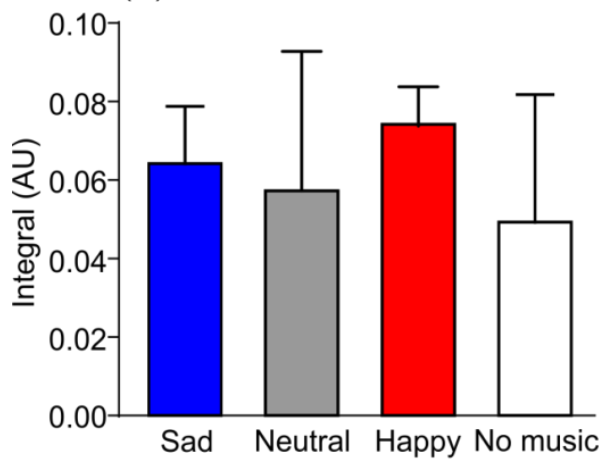

(B) Electrodermal activity

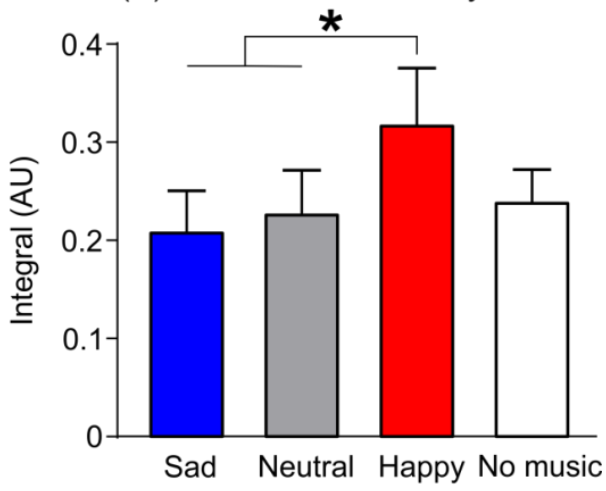

(D) Heart rate variability

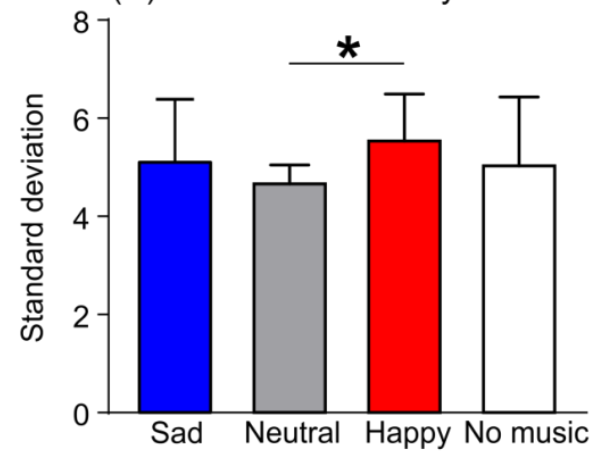

(F) EMG - TA

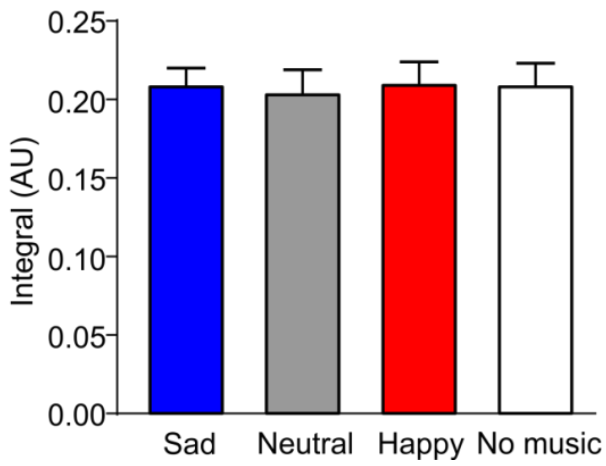

Figure 2: Subjective emotional ratings and physiological data over the conditions.

(A) The subjective emotional ratings are shown, where significant results were found for sad $<$ neutral < happy. (B) For the electrodermal activity, the level was significantly higher during the happy music condition, as compared to sad and neutral. (C) and (D) show the cardiac measures, where no significant effects were found over the conditions for the heart rate, but the heart rate variability in the happy music condition was significantly higher than neutral, respectively. (E) and (F) show the electromyography (EMG) data for the gastrocnemius soleus 
(GS) and tibialis anterior (TA) muscles; no significant effect of condition was found for the EMGs. Median values (with 95\% confidence interval error bars; N.B. box-and-whiskers plot is shown in (A) with the median (line), inter-quartile range (box), and Tukey-corrected error bars (whiskers)) are shown over all the data; ${ }^{*} p<0.05,{ }^{* *} p<0.01$.

These subjective findings were corroborated by changes in the physiological measures. The electrodermal activity showed significant effects of the music (Friedman ANOVA $=16.02, p=$ 0.001; Fig. 2B), where the happy condition showed significantly higher activity than in the sad (Dunn's test $p=0.002$ ) and neutral music conditions (Dunn's test $p=0.029$ ). Although no significant differences were found for the heart rate (Friedman ANOVA $=0.52, p=0.641$; Fig. $2 \mathrm{C}$ ), a significant effect was found for heart rate variability (Friedman ANOVA $=7.82, p=$ 0.048; Fig. 2D), where the happy music condition was significantly higher than neutral (Dunn's test $=0.028$ ). No significant effects of the conditions were found in the EMGs (GS: Friedman ANOVA $=1.47, p=0.690$, Fig. 2E; TA: Friedman ANOVA $=0.222, p=0.895$, Fig. 2F).

For the kinesthetic effects of the music, a significant main effect was observed for the percentage of correct responses (Friedman ANOVA, $F=9.38, p=0.025$; Fig. 3A). Post hoc Dunn's tests revealed that in the sad music condition, the kinesthetic acuity was significantly higher than in the neutral music $(p=0.007)$ and no music $(p=0.032)$. The distribution of the results obtained in each participant over all the conditions, as compared to neutral, are shown in Fig. 3B (Friedman ANOVA $=6.86, p=0.032$ ), where participants in the sad music condition showed significantly improved kinesthetic acuity than in the no music condition (Dunn's test $=0.014$ ). This shows a similar effect that it was the sad emotion, rather than simply listening to music, causing the change in kinesthetic acuity. 


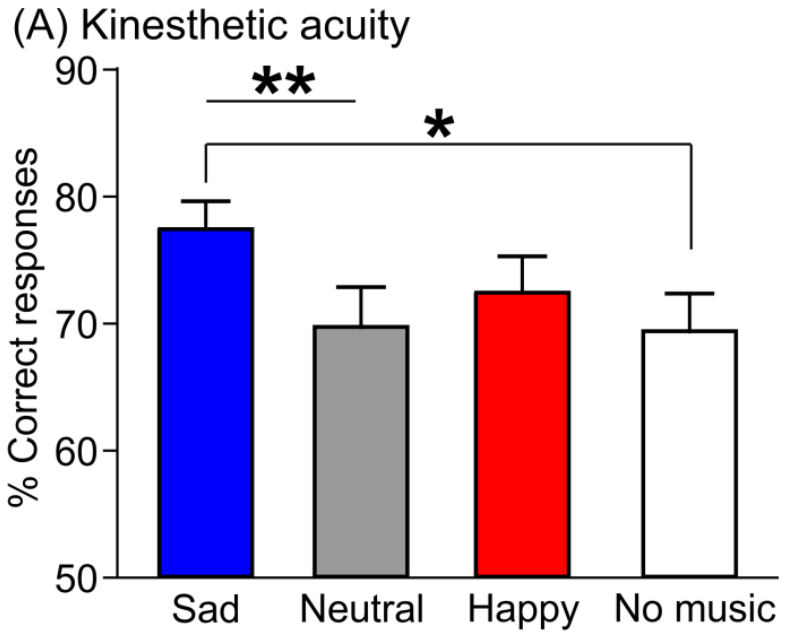

(B) Kinesthetic acuity cf. Neutral

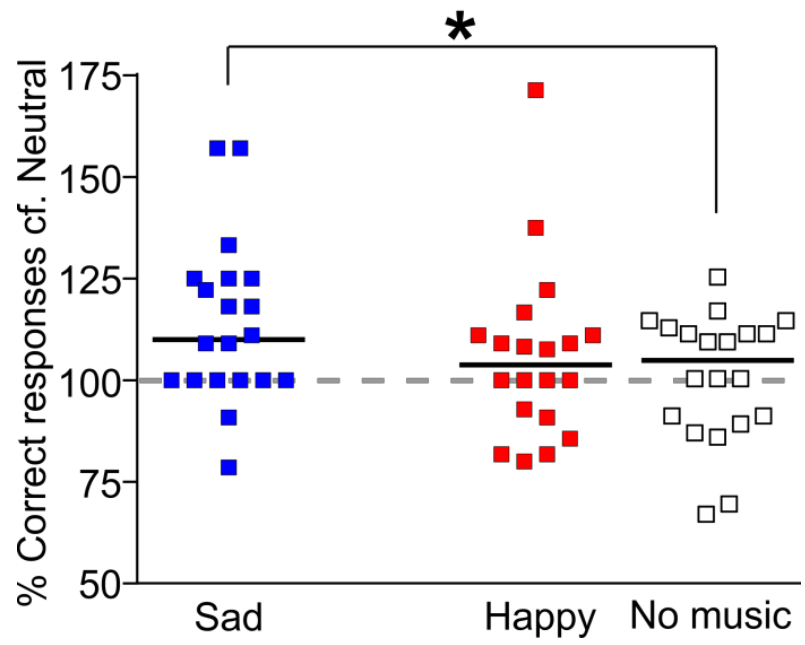

Figure 3: Kinesthetic acuity over the emotional conditions.

(A) The kinesthetic acuity, as measured by the percentage of correct responses, is shown, where there was a significant increase in acuity in the sad music condition, over the neutral and no music conditions. (B) Distribution of the results, by participant, for kinesthetic acuity, as compared to neutral, where the sad music condition showed significantly better acuity than in the no music condition. ${ }^{*} p<0.05, * * p<0.01$.

\section{Discussion}

The present study showed that accuracy in our movement sense depended on our emotional state. More specifically, during feelings of sadness, a significant increase in our ability in detecting movements and reliably reporting their direction was found. On the contrary, happiness was not observed to change our kinesthetic acuity significantly, as compared to the two control conditions (listening to neutral music or no music). The present results are in agreement with the results of our previous microneurographic study [9] where we observed an increased modulation of muscle afferent discharge in response to movement in the sad condition. Such an increase in the change of spindle firing between different phases of a movement may account for the increased perceptual acuity observed presently in the sad condition. Also, no significant change in afferent response was observed in the happy condition, as similarly found here for kinesthetic acuity, all as compared to 
neutral or no music conditions [9]. Therefore, emotions can alter muscle response to movement and this not only impacts the motor activities [13], but also impacts their perception.

In the current experiment and in our previous work [9], the sad condition was most efficient in facilitating, perceptual and neural responses. It is possible that arousal or attentional levels might have been responsible for our observed results. However, the pieces of music were chosen for each participant and were purposely selected to produce higher arousal, making this parameter non-discriminant. In previous studies we demonstrated that attention is a parameter liable to influence the proprioceptive coding of movement $[20,21]$ and likely subsequent movement perception. However, in the present experiment, where participants focused their attention on foot movements, we can consider that this parameter was kept at a similarly high level between the conditions. With regard to the effects we found, feelings of sadness may be in accordance with the situational conditions of participating in an experimental protocol, where the participants were not allowed to move. Along the same lines, the happy condition was incongruent with such an experimental situation, where listening to happy music may trigger excitation and the desire to move, which was not allowed. This experimental limitation may explain why only sad emotion here was efficient at shaping our perception.

Besides changes in the perception of limb movements, some emotional states induced by the music pieces showed differences in the physiological measures. Indeed, the electrodermal activity and heart rate variability were larger during happy emotion, while the impact of sad emotion was less pronounced, as previously reported $[1,13]$. These changes confirm that the music did indeed modify the emotional state of the participants and this was manifest not only in the perception of movement, but in their physiological reaction.

The modulation of our perception by emotional state may be considered a result of central processing, where a direct action from the amygdala may influence sensory cortices [23]. A sensory gain-control mechanism, by means of which sensory stimuli receive increased perceptual processing, has also been demonstrated, notably in the case of visual stimuli [24]. Presently, we show a modulation of perceptual responses to movements, which seems to 
result from a top-down mechanism that shapes the movement information in the periphery (see [9]), which may be used in detecting relevant events and allowing the preparation of appropriate behavioral responses.

\section{References}

[1] T. Brosch, G. Pourtois, D. Sander, The perception and categorisation of emotional stimuli: A review, Cogn. Emot. 24 (2010) 377-400. doi:10.1080/02699930902975754.

[2] T. Brosch, D. Grandjean, D. Sander, K.R. Scherer, Cross-modal Emotional Attention: Emotional Voices Modulate Early Stages of Visual Processing, J. Cogn. Neurosci. 21 (2009) 1670-1679. doi:10.1162/jocn.2009.21110.

[3] U. Proske, S.C. Gandevia, The kinaesthetic senses., J. Physiol. 587 (2009) 4139-46. doi:10.1113/jphysiol.2009.175372.

[4] J.C. Rothwell, M.M. Traub, B.L. Day, J.A. Obeso, P.K. Thomas, C.D. Marsden, Manual motor performance in a deafferented man., Brain. 105 (1982) 515-42.

[5] J.N. Sanes, K.H. Mauritz, M.C. Dalakas, E. V Evarts, Motor control in humans with large-fiber sensory neuropathy., Hum. Neurobiol. 4 (1985) 101-14.

[6] R.L. Sainburg, H. Poizner, C. Ghez, Loss of proprioception produces deficits in interjoint coordination., J. Neurophysiol. 70 (1993) 2136-2147.

[7] M. Hulliger, The mammalian muscle spindle and its central control., Rev. Physiol. Biochem. Pharmacol. 101 (1984) 1-110. doi:10.1007/BFb0027694.

[8] A. Prochazka, Proprioceptive feedback and movement regulation, in: L. Rowell, J. Sheperd (Eds.), Handb. Physiol., American Physiological Society, New York, 1996: pp. 89-127.

[9] R. Ackerley, J.M. Aimonetti, E. Ribot-Ciscar, Emotions alter muscle proprioceptive coding of movements in humans, Sci. Rep. 7 (2017) 8465. doi:10.1038/s41598-01708721-4.

[10] Å. Vallbo, K. Hagbarth, Activity from skin mechanoreceptors recorded percutaneously in awake human subjects., Exp. Neurol. 289 (1968) 270-289.

[11] M.T. Mitterschiffthaler, C.H.Y. Fu, J.A. Dalton, C.M. Andrew, S.C.R. Williams, A functional MRI study of happy and sad affective states induced by classical music, Hum. Brain Mapp. 28 (2007) 1150-1162. doi:10.1002/hbm.20337.

[12] C.H. Hillman, K.S. Rosengren, D.P. Smith, Emotion and motivated behavior: Postural adjustments to affective picture viewing, Biol. Psychol. 66 (2004) 51-62. doi:10.1016/j.biopsycho.2003.07.005.

[13] S.A. Coombes, J.H. Cauraugh, C.M. Janelle, Emotion and movement: Activation of defensive circuitry alters the magnitude of a sustained muscle contraction, Neurosci. Lett. 396 (2006) 192-196. doi:10.1016/j.neulet.2005.11.048.

[14] P.O. Esteves, L.A.S. Oliveira, A.A. Nogueira-Campos, G. Saunier, T. Pozzo, J.M. Oliveira, E.C. Rodrigues, E. Volchan, C.D. Vargas, Motor planning of goal-directed action is tuned by the emotional valence of the stimulus: a kinematic study, Sci. Rep. 6 (2016) 28780. doi:10.1038/srep28780.

[15] U. Windhorst, Muscle proprioceptive feedback and spinal networks, Brain Res. Bull. 73 (2007) 155-202. doi:10.1016/j.brainresbull.2007.03.010. 
[16] S.C. Gandevia, D. Burke, Effect of training on voluntary activation of human fusimotor neurons, J. Neurophysiol. 54 (1985) 1422-1429.

[17] A.M. van Loon, W.P.M. van den Wildenberg, A.H. van Stegeren, K.R. Ridderinkhof, G. Hajcak, Emotional stimuli modulate readiness for action: A transcranial magnetic stimulation study, Cogn. Affect. Behav. Neurosci. 10 (2010) 174-181. doi:10.3758/CABN.10.2.174.

[18] E. Ribot-Ciscar, V. Hospod, J.-M. Aimonetti, Noise-enhanced kinaesthesia: a psychophysical and microneurographic study., Exp. Brain Res. 228 (2013) 503-11. doi:10.1007/s00221-013-3581-6.

[19] K.M. Refshauge, R.C. Fitzpatrick, Perception of movement at the human ankle: effects of leg position., J. Physiol. 488 ( Pt 1) (1995) 243-8.

[20] V. Hospod, J. Aimonetti, J.P. Roll, E. Ribot-Ciscar, Changes in human muscle spindle sensitivity during a proprioceptive attention task., J. Neurosci. 27 (2007) 5172-8. doi:10.1523/JNEUROSCI.0572-07.2007.

[21] E. Ribot-Ciscar, V. Hospod, J.P. Roll, J. Aimonetti, Fusimotor drive may adjust muscle spindle feedback to task requirements in humans., J. Neurophysiol. 101 (2009) 63340. doi:10.1152/jn.91041.2008.

[22] S. Khalfa, M. Roy, P. Rainville, S. Dalla Bella, I. Peretz, Role of tempo entrainment in psychophysiological differentiation of happy and sad music?, Int. J. Psychophysiol. 68 (2008) 17-26. doi:10.1016/j.ijpsycho.2007.12.001.

[23] T. Brosch, K.R. Scherer, D. Grandjean, D. Sander, The impact of emotion on perception, attention, memory, and decision-making, Swiss Med. Wkly. 143 (2013) w13786. doi:10.4414/smw.2013.13786.

[24] T. Brosch, D. Sander, G. Pourtois, K.R. Scherer, Beyond fear: rapid spatial orienting toward positive emotional stimuli., Psychol. Sci. 19 (2008) 362-70. doi:10.1111/j.1467-9280.2008.02094.x.

Declarations of interest: none

Author contributions: All authors conceived and designed the research; L.S.-A. performed the experiments; L.S.-A., R.A. and E.R.-C. analyzed the data; all authors interpreted the results of the experiments, prepared the figures, and drafted the manuscript. All authors have approved the final article. 